\title{
AUTOEFICACIA Y RIESGO PSICOSOCIAL EN PERSONAL TÉCNICO DE UN SERVICIO DE ADOPCIONES
}

\author{
Sergio Sánchez-Sevilla \\ Rocío Guil Bozal \\ Serafín Cruces Montes \\ Antonio Zayas García \\ Departamento de Psicología. \\ Universidad de Cádiz.sergio.sanchez@uca.es \\ https://doi.org/10.17060/ijodaep.2017.n1.v3.1002
}

Fecha de Recepción: 27 Febrero 2017

Fecha de Admisión: 1 Abril 2017

\section{RESUMEN}

Es necesario que la Psicología siga dedicando esfuerzos a elaborar mapas de riesgos psicosociales por ocupaciones, así como a facilitar la comprensión de las relaciones entre riesgos y consecuencias, comprendiendo también el papel de los factores de vulnerabilidad y protección. Este estudio aporta como novedad la evaluación de riesgos psicosociales en un colectivo hasta ahora poco estudiado: Ios técnicos y técnicas de un servicio de información, formación, valoración de idoneidad y seguimientos postadoptivos en procedimientos de adopciones internacionales. Los objetivos de esta investigación son: determinar la proporción de profesionales expuestos a condiciones de trabajo de riesgo psicosocial; establecer el nivel de autoeficacia de estos profesionales para trabajar en condiciones de riesgo psicosocial; determinar la relación entre el nivel de exposición a riesgos psicosociales y el nivel de autoeficacia de estos profesionales. Se llevó a cabo un estudio transversal mediante encuesta en el que participó una muestra de 51 técnicos y técnicas, de un total de 72 personas que componían dicho servicio en la comunidad autónoma de Andalucía. Los instrumentos utilizados, además de un formulario de datos sociodemográficos, fueron el Inventario de Riesgos Psicosociales (León y Avargues, 2004) y la Escala de Autoeficacia Profesional bajo condiciones de Riesgo Psicosocial (Sánchez-Sevilla, Guillén y León-Rubio, 2006). Los resultados pusieron de manifiesto que casi la mitad de las personas evaluadas se encuentran expuestas a condiciones laborales de mucha tensión que suponen un riesgo para la calidad de vida laboral y la salud relacionada con el estrés laboral. En lo referente a la autoeficacia profesional bajo condiciones de riesgo psicosocial, el conjunto de la muestra obtuvo una puntuación moderada-alta. Con respecto a la posible relación de la autoeficacia con el nivel de riesgo psicosocial soportado por los profesionales evaluados, se encontró una relación inversa entre ambas variables.

PALABRAS CLAVES: riesgos psicosociales, estrés laboral, autoeficacia, adopciones 


\section{AUTOEFICACIA Y RIESGO PSICOSOCIAL EN PERSONAL TÉCNICO DE UN SERVICIO DE ADOPCIONES}

\section{ABSTRACT}

Self-efficacy and psychosocial risks in technical staff of an adoption service.

It is necessary that Psychology continues to devote efforts to map psychosocial risks in different occupations, as well as to facilitate the understanding of the relationship between risks and consequences, including the role of vulnerability and protective factors. This study brings as a novelty the evaluation of psychosocial risks in a group that has not been studied until now: the technical staff of a service of information, training, assessment of suitability and post-adoption follow-ups in international adoption procedures. The objectives of this research are: to determine the proportion of professionals exposed to psychosocial risk working conditions; to establish the level of self-efficacy of these professionals for working under conditions of psychosocial risk; to establish the relationship between the degree of exposure to psychosocial risks and the level of self-efficacy of these professionals. A cross-sectional study was carried out through a survey in which a sample of 51 technicians participated, out of a total of 72 people who composed the service in the Autonomous Community of Andalusia. The instruments used, in addition to a sociodemographic data form, were the Inventory of Psychosocial Risks (León \& Avargues, 2004) and the Professional Self-Efficacy under Psychosocial Risk Conditions Scale (Sánchez-Sevilla, Guillén \& León-Rubio, 2006). The results showed that almost half of the people evaluated are exposed to very stressful working conditions that are a risk to work life quality and health related to work stress. Regarding professional self-efficacy under psychosocial risk conditions, the sample as a whole had a moderate-high score. On the possible relationship of self-efficacy with the level of psychosocial risk borne by the professionals evaluated, an inverse relationship was found between both variables.

KEY WORDS: psychosocial risks, work stress, self-efficacy, adoptions

\section{ANTECEDENTES}

Si bien la literatura acerca del fenómeno adoptivo es abundante, la mayor parte se aproxima al tema dirigiendo la mirada especialmente hacia los futuros padres/madres adoptantes, hacia los futuros hijos o hijas adoptivos, a los procesos psicosociales familiares que se dan en dicho proceso, a los modelos de intervención profesional 0 al entramado normativo y legal en torno a la adopción. Sin embargo, son escasas las investigaciones y la literatura centradas en las personas que desarroIlan su labor profesional en este ámbito de actuación, sobre sus condiciones de trabajo, los riesgos laborales (básicamente psicosociales) a los que están expuestos y su impacto sobre la salud y la calidad de vida laboral.

Es necesario que la Psicología siga dedicando esfuerzos a elaborar mapas de riesgos psicosociales por ocupaciones, así como a facilitar la comprensión de las relaciones entre riesgos y consecuencias, comprendiendo también el papel de los factores de vulnerabilidad y protección. Este estudio aporta como novedad la evaluación de riesgos psicosociales en un colectivo hasta ahora poco estudiado: Ios técnicos y técnicas del "Servicio de información, formación, valoración de idoneidad y seguimientos postadoptivos en procedimientos de adopciones internacionales" de Andalucía.

Los factores psicosociales son las condiciones presentes en una situación laboral directamente relacionadas con la organización del trabajo, con el contenido del puesto, con la realización de la tarea e incluso con el entorno, que tienen la capacidad de afectar al desarrollo del trabajo y a la salud de las personas trabajadoras (Gil-Monte, 2009). Cuando esos factores son desfavorables y tienen consecuencias perjudiciales sobre la salud y el bienestar, decimos que se han convertido en factores de riesgo psicosocial o fuentes de estrés laboral.

La investigación ha permitido establecer de forma fiable relaciones entre el estrés laboral y la disminución del rendimiento y la productividad, la falta de control sobre las tareas y problemas en 
la salud y el bienestar de los trabajadores (Ares-Camerino et al., 2010; Edwards, Cockerton, y Guppy, 2007; Guillén, León, Gala, Sánchez-Sevilla y Ramos, 2005; Karasek y Theorell, 1990; Maslach, Schaufeli y Leiter, 2001; Vladut y Kallay, 2010).

El modelo de estrés laboral de demanda-control-apoyo (Karasek y Theorell, 1990) es uno de los más influyentes en el área de salud laboral, ya que se ha utilizado como base teórica para la investigación y está entre los modelos que han sido probados empíricamente más frecuentemente (GilMonte, 2010; Luchman y González-Morales, 2013), Este modelo define las situaciones de riesgo en base a tres dimensiones de las condiciones de trabajo: demandas del trabajo, control del individuo y apoyo social.

El modelo de Karasek de "tensión laboral" no sólo establece que la combinación de elevadas demandas y bajo margen de decisión y apoyo social llevará a resultados negativos de salud, sino que, además, el modelo contiene importantes predicciones en cuanto a la socialización de rasgos de personalidad, patrones de conducta y competencias relacionadas con en el trabajo.

En este sentido, entre las variables personales más relevantes por su papel en la etiología y afrontamiento del estrés laboral, destaca la autoeficacia. Bandura (1997), desde su Teoría Social Cognitiva, define la autoeficacia como las creencias en las propias capacidades para organizar y ejecutar los cursos de acción requeridos que producirán determinados logros o resultados, lo cual resulta fundamental para controlar las demandas del entorno.

La autoeficacia profesional sería la responsable de que los profesionales no percibieran las situaciones como amenazantes y estresantes, facilitando al mismo tiempo el afrontamiento exitoso de las mismas. Por el contrario, cuando no se alcanzan sentimientos de competencia y eficacia profesional aparecen altos niveles de estrés laboral y, en algunos casos, se manifiesta el síndrome de burnout (Grau, Salanova y Peiro, 2012; Madden-Szeszko, 2000; Salanova, Grau y Martinez, 2005; Sánchez-Sevilla, Guillén y León-Rubio, 2006; Yu et al., 2015).

No obstante, sigue siendo necesario ampliar la investigación sobre la relación entre la autoeficacia y los riesgos psicosociales, dadas las inconsistencias que a este respecto pone de manifiesto la literatura (Peng, Schaubroeck y Xie, 2015). Uno de los principales motivos de las inconsistencias encontradas es que muchas investigaciones han utilizado medidas de autoeficacia general en lugar de utilizar medidas de autoeficacia profesional o de autoeficacia específica para el afrontamiento de eventos estresantes (Bandura, 1997; Godoy-Izquierdo y Godoy, 2006).

En esta investigación queremos contribuir al esclarecimiento de estas relaciones entre los riesgos psicosociales y una autoeficacia específica: la autoeficacia profesional bajo condiciones de riesgo psicosocial.

\section{OBJETIVOS}

Los objetivos operativos de esta investigación son:

Determinar la proporción de profesionales de los servicios de adopciones expuestos a condiciones de trabajo de riesgo psicosocial

Establecer el nivel de autoeficacia de estos profesionales para trabajar en condiciones de riesgo psicosocial

Determinar la relación entre el nivel de exposición a riesgos psicosociales y el nivel de autoeficacia de estos profesionales

\section{MUESTRA}

La población objeto de nuestro estudio es el colectivo de técnicos del "Servicio de información, formación, valoración de idoneidad y seguimientos postadoptivos en procedimientos de adopciones 


\section{AUTOEFICACIA Y RIESGO PSICOSOCIAL EN PERSONAL TÉCNICO DE UN SERVICIO DE ADOPCIONES}

internacionales" para la comunidad autónoma andaluza. La muestra final estuvo compuesta por 52 sujetos, de un total de 72 personas que componían dicho Servicio en el momento del estudio, 10 cual supone un $72,22 \%$ de la población total. El 90,4\% fueron mujeres (47 sujetos) y el 9,6\% fueron hombres (5 sujetos). El rango de edad mayoritario es el comprendido entre los 31 y los 40 años, con un $55,8 \%$ (29 casos). Con respecto a los estudios académicos de los profesionales encuestados, el 55,8\% (29 sujetos) posee una Licenciatura en Psicología, mientras que el 44,2\% (23 sujetos) ha cursado una Diplomatura en Trabajo Social. Con respecto a la antigüedad en el puesto de trabajo, el 92,3\% (48 sujetos) acumula más de 2 años de antigüedad.

\section{METODOLOGÍA}

Se llevó a cabo un estudio transversal mediante encuesta. Para la recogida de datos se elaboró un cuadernillo que contenía los siguientes instrumentos:

- Registro de Variables Personales y de Condiciones de Trabajo

Es un autoinforme de elaboración propia, sin estructura psicométrica, donde se recogen las siguientes variables sociodemográficas y laborales: género, edad, antigüedad en el desempeño del puesto y estudios realizados.

- Inventario de Riesgos Psicosociales.

Elaborado por León y Avargues (2004) con la finalidad de detectar indicadores de riesgo de carácter psicosocial en el puesto de trabajo, para posteriormente diseñar un plan de actuación preventiva bien fundamentado. A lo largo de 30 ítems, los factores psicosociales se agrupan en tres dimensiones, siguiendo el modelo de demanda-control de Karasek: demandas del trabajo, control del trabajo y apoyo social en el trabajo.

- Escala de Autoeficacia Profesional bajo condiciones de Riesgo Psicosocial.

Elaborada por Sánchez-Sevilla et al. (2006). Evalúa el grado de confianza 0 de seguridad que tiene un profesional en poder hacer su trabajo bajo diversas circunstancias adversas consideradas por la literatura como situaciones o condiciones de riesgo laboral psicosocial (v.g., "Si las instalaciones o el mobiliario son incómodos", "Si me encargan un trabajo importante en el que cualquier error sería grave", "Si no tengo la posibilidad de participar en la toma de decisiones", "Cuando tengo que tratar con personas difíciles", etc.). Los sujetos deben contestar a cada ítem asignando una puntuación en una escala de confianza entre 0 (Incapaz de poder hacerlo) y 100 (Seguro de poder hacerlo).

\section{RESULTADOS}

A partir de la combinación de las tres dimensiones (demandas, control y apoyo social) del Inventario de Riesgos Psicosociales se han generado tres situaciones psicosociales (bajo riesgo, riesgo moderado y alto riesgo), con sus correspondientes implicaciones en el terreno del riesgo de tensión psicológica y enfermedad. Concretamente, basándonos en lo establecido por Karasek y Theorell (1990), hemos considerado que la combinación de una alta demanda con un bajo control sobre el trabajo constituye una situación de "riesgo moderado". Si bien hay estudios que consideran esta combinación como de "alta tensión o alto riesgo", nosotros hemos querido aplicar un criterio más restrictivo y reservar esta calificación de "alto riesgo" sólo para aquellos casos en que se cumpliese una tercera condición adicional: concretamente un bajo apoyo social.

Según esto, la evaluación del grado de exposición a condiciones de riesgo psicosocial de los sujetos de nuestra muestra arrojó los siguientes resultados: el 53,8\% de las personas encuestadas trabaja en condiciones de bajo riesgo, el $28,8 \%$ soporta un nivel moderado de riesgo y el $17,3 \%$ está sometida a condiciones de alto riesgo psicosocial (ver Figura 1). 
Figura 1:

Distribución de porcentajes según niveles para la variable "Riesgo psicosocial"

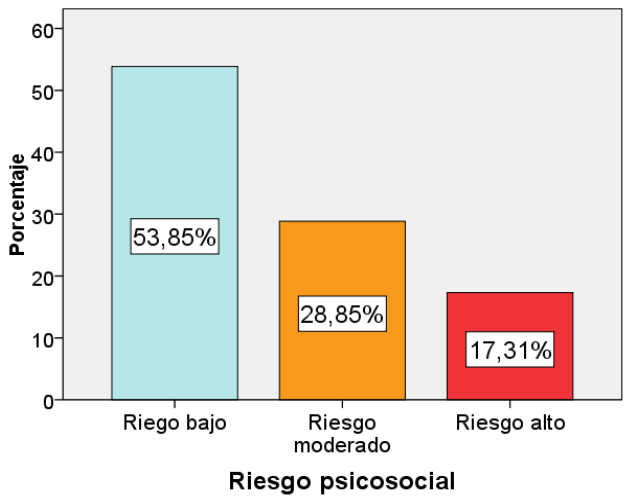

Si se suma el porcentaje de sujetos en situación de riesgo (ya sea alto o moderado), se puede comprobar que casi la mitad de las personas encuestadas se encuentran expuestas a condiciones laborales de mucha tensión que suponen un riesgo de enfermedad relacionada con el estrés laboral.

En lo referente a la autoeficacia profesional bajo condiciones de riesgo psicosocial, el conjunto de la muestra obtuvo una puntuación media de 67,4 (DT.=11,2), con una mínima de 38,3 y una máxima de 88,3 . Viendo la curva, y teniendo en cuenta que en el instrumento de medida utilizado los sujetos debían contestar en una escala de 0 a 100, se puede considerar que la puntuación media obtenida es moderada con tendencia a ligeramente alta (ver Figura 2).

Figura 2:

Histograma de frecuencias para la variable "Autoeficacia"

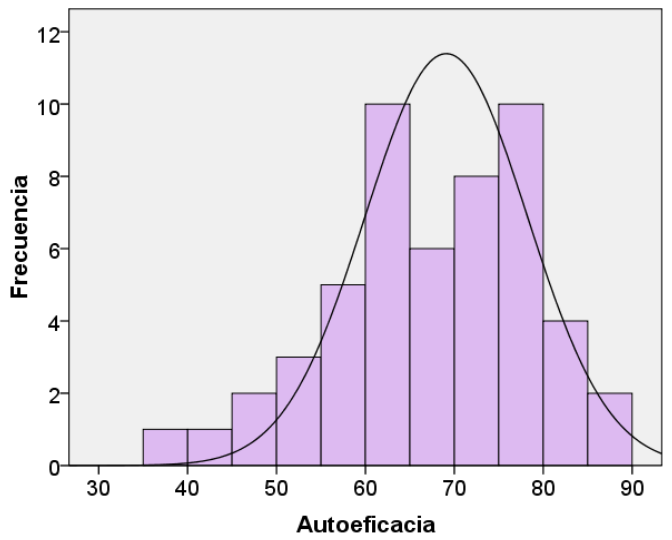




\section{AUTOEFICACIA Y RIESGO PSICOSOCIAL EN PERSONAL TÉCNICO DE UN SERVICIO DE ADOPCIONES}

Con respecto a la posible relación entre el nivel de riesgo psicosocial soportado por los profesionales evaluados y la variable autoeficacia, se encontró una asociación estadísticamente significativa (ver Tablas 1, 2 y 3).

Tabla 1:

Descriptivos de Autoeficacia * Nivel de riesgo psicosocial

\section{Descriptivos}

Autoeficacia

\begin{tabular}{|c|c|c|c|c|c|c|c|c|}
\hline & \multirow[b]{2}{*}{$N$} & \multirow[b]{2}{*}{ Media } & \multirow[b]{2}{*}{$\begin{array}{c}\text { Desviación } \\
\text { típica }\end{array}$} & \multirow[b]{2}{*}{ Error típico } & \multicolumn{2}{|c|}{$\begin{array}{l}\text { Intervalo de confianza } \\
\text { para la media al } 95 \%\end{array}$} & \multirow[b]{2}{*}{ Minimo } & \multirow[b]{2}{*}{ Máximo } \\
\hline & & & & & $\begin{array}{l}\text { Limite } \\
\text { inferior }\end{array}$ & $\begin{array}{l}\text { Limite } \\
\text { superior }\end{array}$ & & \\
\hline Riego bajo & 28 & 70,4563 & 8,46339 & 1,59943 & 67,1746 & 73,7381 & 53,89 & 88,33 \\
\hline $\begin{array}{l}\text { Riesgo } \\
\text { moderado }\end{array}$ & 15 & 66,2407 & 12,99541 & 3,35540 & 59,0441 & 73,4374 & 43,33 & 82,22 \\
\hline Riesgo alto & 9 & 59,8765 & 12,58851 & 4,19617 & 50,2002 & 69,5529 & 38,33 & 75,56 \\
\hline Total & 52 & 67,4092 & 11,16473 & 1,54827 & 64,3009 & 70,5175 & 38,33 & 88,33 \\
\hline
\end{tabular}

Tabla 2:

ANOVA de Autoeficacia * Nivel de riesgo psicosocial

\begin{tabular}{lrrrrr}
$\begin{array}{l}\text { ANOVA } \\
\text { Autoeficacia }\end{array}$ & \multicolumn{1}{c}{ Sumade } & & & & \\
\hline & cuadrados & gl & cuadrática & F & Sig. \\
\hline Inter-grupos & 791,131 & 2 & 395,566 & 3,482 &, 039 \\
Intra-grupos & 5566,079 & 49 & 113,593 & & \\
Total & 6357,210 & 51 & & & \\
\hline
\end{tabular}

Tabla 3:

Pruebas post hoc de Autoeficacia * Nivel de riesgo psicosocial

Comparaciones múltiples

Variable dependiente : Autoeficacia

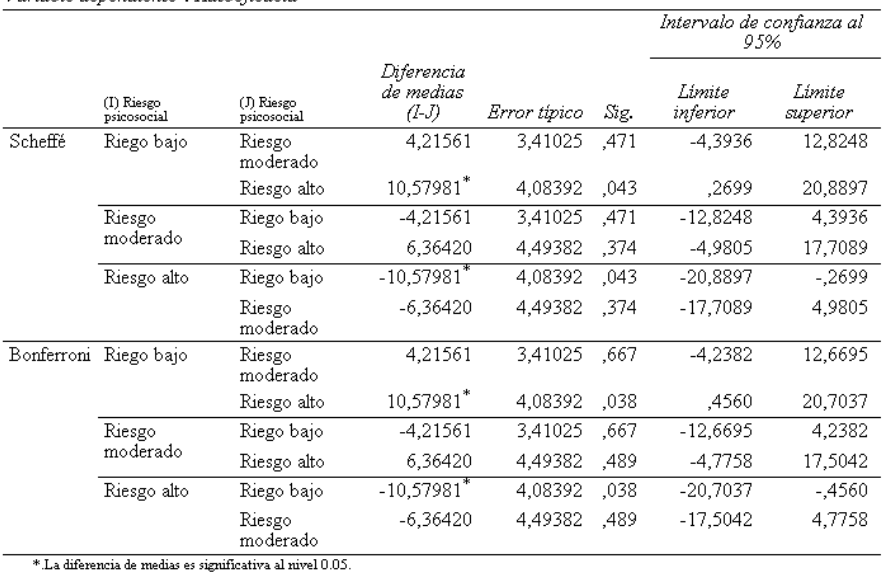


Se puede observar que existe una diferencia significativa entre las puntuaciones de autoeficacia de los sujetos en situación de bajo riesgo psicosocial y las de los sujetos en riesgo alto. Concretamente, la autoeficacia para trabajar en condiciones de riesgo psicosocial es significativamente más alta en los sujetos del grupo de riesgo bajo que en los de riesgo alto y viceversa (ver Figura 3).

Figura 3:

Línea de medias de Autoeficacia * Riesgo psicosocial

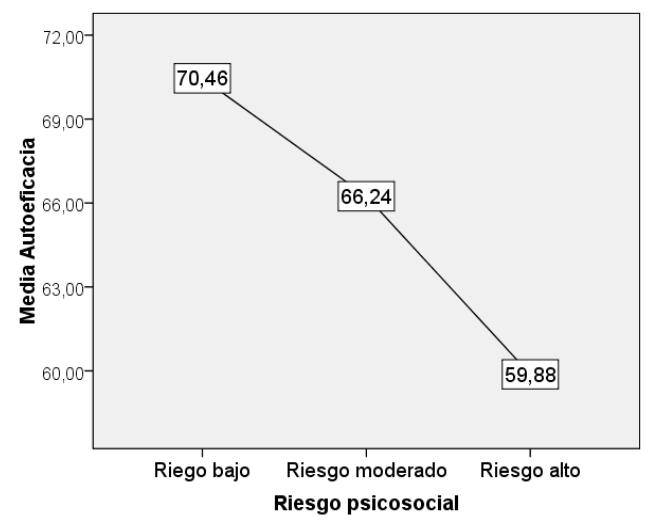

Por último, se realizó una regresión múltiple para predecir la autoeficacia profesional en función del género, la edad, la antigüedad en el puesto de trabajo, el tipo de estudios cursados y el riesgo psicosocial. El modelo de regresión múltiple predijo estadísticamente la autoeficacia profesional, $\mathrm{F}$ $(5,46)=2,587, p<0,05, R^{2}$ ajustado $=0,135$. De las cuatro variables, sólo el nivel de riesgo psicosocial aportó estadísticamente a la predicción, $p<0,05$. Los coeficientes de regresión y los errores estándar se pueden encontrar en la Tabla 4.

Tabla 4:

Coeficientes del modelo de regresión múltiple

Coeficientes $^{a}$

\begin{tabular}{|c|c|c|c|c|c|c|c|c|}
\hline \multirow[b]{2}{*}{ Modelo } & & \multicolumn{2}{|c|}{$\begin{array}{l}\text { Coeficientes no } \\
\text { estandarizados }\end{array}$} & \multicolumn{3}{|l|}{$\begin{array}{c}\text { Coeficientes } \\
\text { estandariza } \\
\text { dos }\end{array}$} & \multicolumn{2}{|c|}{$\begin{array}{c}95,0 \% \text { intervalo de } \\
\text { confianza para } B\end{array}$} \\
\hline & & $B$ & $\begin{array}{l}\text { Error } \\
\text { estándar }\end{array}$ & Beta & $t$ & Sig. & $\begin{array}{l}\text { Limite } \\
\text { inferior }\end{array}$ & $\begin{array}{l}\text { Limite } \\
\text { superior }\end{array}$ \\
\hline 1 & (Constante) & 75,401 & 13,122 & & 5,746 &, 000 & 48,988 & 101,814 \\
\hline & Género & 6,707 & 4,954 &, 181 & 1,354 &, 182 & $-3,264$ & 16,678 \\
\hline & Edad & 2,232 & 2,171 &, 136 & 1,028 & ,309 & $-2,138$ & 6,601 \\
\hline & $\begin{array}{l}\text { Antiguedad en } \\
\text { el puesto }\end{array}$ & $-8,426$ & 5,381 &,- 206 & $-1,566$ &, 124 & $-19,257$ & 2,406 \\
\hline & Estudios & 2,833 & 2,896 &, 129 & ,978 & ,333 & $-2,995$ & 8,662 \\
\hline & $\begin{array}{l}\text { Riesgo } \\
\text { psicosocial }\end{array}$ & $-4,780$ & 1,931 &,- 333 & $-2,475$ &, 017 & $-8,668$ &,- 893 \\
\hline
\end{tabular}

a. Variable dependiente: Autoeficacia 


\section{AUTOEFICACIA Y RIESGO PSICOSOCIAL EN PERSONAL TÉCNICO DE UN SERVICIO DE ADOPCIONES}

\section{DISCUSIÓN Y CONCLUSIONES}

Los resultados sobre riesgo psicosocial, en base a los niveles de demanda, control y apoyo experimentados por los sujetos objeto de estudio, en los que casi la mitad de los técnicos quedan situados dentro de los grupos de alto y moderado riesgo psicosocial, si bien hasta ahora no habían sido cuantificados a través de una evaluación de riesgos psicosociales, sí habían sido avanzados por la literatura al identificar situaciones y condiciones de trabajo que suponen un desafío para la praxis de los profesionales de los servicios de adopciones. Por ejemplo, diversos autores hablan de que las distintas fases de la intervención profesional aparecen frecuentemente como desconectadas, de la falta de homogeneidad entre los distintos técnicos en cuanto a su nivel de especialización en adopción, de la necesidad de la formación permanente del personal técnico, de la complejidad del trabajo en equipos multidisciplinares, de la alta exigencia y ansiedad de las familias solicitantes y los conflictos que se generan con las mismas, de las dificultades de adaptación familiar en la fase postadoptiva, de las presiones institucionales, etc. (Berástegui, 2008; Burgos y Legido, 2008; Fernández Molina y Alarcón, 2015; Galli y Viero, 2007; León, Sánchez-Sandoval, Palacios y Román, 2010; López y Marmolejo, 2008; Palacios, 2008; Sánchez-Sandoval, León y Román, 2012).

En cuanto a la autoeficacia profesional bajo condiciones de riesgo psicosocial, los resultados arrojaron niveles medios-altos en la muestra estudiada. Ello puede tener su explicación en que el trabajo dentro del ámbito adoptivo implica una praxis profesional muy específica, cuyas funciones, objetivos, criterios y direccionalidad han tenido un apoyo fundamental, tras la implantación y desarrollo de un modelo y manual de intervención específico (Palacios, 2008), así como el apoyo normativo que se ha ido desarrollando al respecto desde la creación del Servicio en Andalucía. A esto habría que sumar la hipótesis sostenida por algunos autores (Harrison, 1983; Madden-Szeszko, 2000) de que las expectativas de autoeficacia son mayores en las profesiones de servicio o ayuda, donde prevalece una filosofía humanista del trabajo con una fuerte motivación vocacional y elevados sentimientos prosociales, actuando como un elemento protector ante los riesgos psicosociales.

Con respecto a esta relación entre la autoeficacia y el riesgo psicosocial, nuestros resultados evidenciaron mayores niveles de autoeficacia profesional en los sujetos pertenecientes al grupo de bajo riesgo psicosocial que en los sujetos que trabajan en condiciones de alto riesgo. El carácter autorregulador de la eficacia percibida condiciona que aquellas personas que se juzgan a sí mismas como ineficaces en el afrontamiento de las demandas ambientales valoren de más sus propias deficiencias, así como las dificultades potenciales a las que tendrán que hacer frente (Bandura, 1982). A su vez, en el caso de la autoeficacia específica para el afrontamiento en situaciones de estrés, ésta tendría una influencia decisiva en los esfuerzos adaptativos que la persona pone en marcha para responder a las situaciones que son interpretadas como demandantes (Guillén y Sánchez-Sevilla, 2012), influyendo especialmente en algunos de los procesos implicados, especialmente en la evaluación secundaria (evaluación que la persona hace de sus capacidades personales para responder eficazmente a las demandas) y la selección y ejecución de las estrategias de afrontamiento (GodoyIzquierdo et al., 2008).

A este respecto, los resultados de nuestro modelo de regresión parecen contribuir a los hallazgos de otras investigaciones (Friedman, 2000; García-Renedo, Llorens, Cifre y Salanova, 2006; GilMonte y García-Juesas, 2008; Llorens, Schaufeli, Bakker y Salanova, 2007; Mackay y Cooper, 1987) que ponen de manifiesto el carácter cíclico-bidireccional de las relaciones entre autoeficacia y riesgo psicosocial. Es decir, la autoeficacia puede aumentar como consecuencia del afrontamiento exitoso del estrés laboral o puede disminuir como consecuencia de la exposición prolongada a una situación laboral estresante. Por ejemplo, dado que la autoeficacia es un conjunto de creencias en continua revisión y evaluación, una persona que desarrolle estrés laboral o burnout, experimentaría 
sentimientos de baja realización personal en el trabajo, un incremento del agotamiento emocional, 0 actitudes de despersonalización y distanciamiento de su trabajo, que ocasionarían que, cuando el profesional volviese a evaluar sus condiciones de trabajo, la percepción de la dificultad de las demandas se viese incrementada y la percepción del control y del apoyo social en su trabajo se viera disminuida.

Las implicaciones de este trabajo de investigación con respecto a la prevención de riesgos laborales se nos antojan interesantes, ya que, considerando que la incidencia de los riesgos psicosociales es importante en profesionales que prestan una labor de servicio social, como es el caso del personal técnico de los servicios de adopciones, el deterioro de su calidad de vida laboral también conlleva perjuicios para los usuarios de los servicios y sobre la sociedad en general (Gil-Monte y Peiró, 1999).

Por eso, tomando como base los resultados obtenidos en esta investigación, se pueden proponer medidas de prevención de riesgos psicosociales que, siguiendo las recomendaciones de diversos autores, deberían actuar en tres planos: organizacional, interpersonal-grupal, y personal (Guerrero y Vicente, 2002; Rodríguez, González-Morales y Carbonell, 2007; Sánchez-Sevilla, Cruces, Guil y Guillén, 2012).

Como es lógico, las estrategias organizativas tendrían por objetivo eliminar o reducir el impacto de los factores de la organización del trabajo e institucionales que suponen un riesgo para los técnicos del servicio de adopciones.

En el plano interpersonal y grupal se recomiendan los programas orientados a potenciar las relaciones interpersonales en los equipos de trabajo multidisciplinar, la resolución de conflictos interpersonales y la creación de redes de apoyo social de carácter profesional.

En el plano personal, se recomienda que las intervenciones se orienten a que el personal técnico adquiera estrategias de afrontamiento activas, sí como la capacidad de gestión del pensamiento autorreferente como medio para desarrollar creencias de autoeficacia profesional.

En cuanto a las limitaciones de este estudio y las recomendaciones de cara a futuras investigaciones, las principales son las que comparten todas las investigaciones basadas en medidas de autoinforme, o las relacionadas con la cautela al establecer relaciones causales a partir de diseños transversales. Por otra parte, algunas variables de tipo socio-demográfico y laboral apuntaron tendencias que no pudieron ser consideradas significativas estadísticamente, por lo que sería conveniente ampliar este estudio con nuevos datos a partir de una muestra de mayor tamaño, por ejemplo, ampliando el estudio a otros Servicios de Adopciones de otras comunidades autónomas.

\section{REFERENCIAS BIBLIOGRÁFICAS}

Ares-Camerino, A., Guillén-Gestoso, C., Sánchez-Sevilla, S., Suárez-Collantes, M., Sainz-Vera, B., \& Soto-Pino, M. L. (2010). Estrés laboral. Prevalencia en una corporación local. Revista de la Sociedad Española de Medicina y Seguridad del Trabajo, 5(4), 194-201.

Bandura, A. (1997). Self-efficacy: The exercise of control. New York: Freeman.

Bandura, A. (1982). Self-efficacy mechanism in human agency. American Psychologist, 37, 122147.

Berástegui, A. (2008). La valoración de la idoneidad de los candidatos a la adopción: reflexiones y sugerencias desde la investigación. En C. Pérez Testor (Ed.), Postadopción y vínculo familiar (pp. 101-117). Barcelona: Universidad Ramón Lull.

Burgos, A., \& Legido, M. (2008). El Servicio de Protección de Menores y el Procedimiento de Adopción. En R. Martínez \& J. M. Gómez (Eds.), La adopción de menores: Retos y necesidades (pp. 93-125). Sevilla: Asociación Andaluza de Ayuda a la Adopción y la Infancia (LLAR). 


\section{AUTOEFICACIA Y RIESGO PSICOSOCIAL EN PERSONAL TÉCNICO DE UN SERVICIO DE ADOPCIONES}

Edwards, J. A., Cockerton, T., \& Guppy, A. (2007). A longitudinal study examining the influence of work and non-work stressors upon well-being: A multi-group analysis. International Journal of Stress Management, 14(3), 294-311.

Fernández Molina, M., \& Alarcon, E. (2015). Adoption and Homosexual Parenting. Professionals' Attitudes in a Spanish Sample. Acción Psicológica, 12(1), 91-102.

Friedman, I. A. (2000). Burnout in teachers: Shattered dreams of impeccable professional performance. Journal of Clinical Psychology, 56(5), 595-606.

Galli, J., \& Viero, F. (2007). El fracaso en la adopción: Prevención y reparación. Madrid: Grupo 5.

García-Renedo, M., Llorens, S., Cifre, E., \& Salanova, M. (2006). Antecedentes afectivos de la autoeficacia docente: Un modelo de relaciones estructurales. Revista de Educación, 339, 387-400

Gil-Monte, P. R. (2009). Algunas razones para considerar los riesgos psicosociales en el trabajo y sus consecuencias en la salud pública. Revista Española de Salud Pública, 83(2), 169-173.

Gil-Monte, P. (2010). Situación actual y perspectiva de futuro en el estudio del estrés laboral: La Psicología de la Salud Ocupacional. Informació Psicològica, 100, 68-83.

Gil-Monte, P. R., \& García-Juesas, J. A. (2008). Efectos de la sobrecarga laboral y la autoeficacia sobre el síndrome de quemarse por el trabajo (burnout). Un estudio longitudinal en enfermería. Revista Mexicana de Psicología, 25, 329-337

Gil-Monte, P. R., \& Peiró, J. M. (1999). Perspectivas teóricas y modelos interpretativos para el estudio del síndrome de quemarse por el trabajo. Anales de Psicología, 15(2), 261-268.

Godoy-Izquierdo, D., \& Godoy, J.F. (2006). Escala de Autoeficacia Específica para el Afrontamiento del Estrés (EAEAE). En V. E. Caballo (Ed.): Manual para la evaluación clínica de los trastornos psicológicos (en cd adjunto a la obra). Madrid: Pirámide.

Godoy-Izquierdo, D., Godoy García, J. F., López-Chicheri Garcia, I., Martínez Delgado, A., Gutiérrez Jiménez, S., \& Vázquez Vázquez, L. (2008). Propiedades psicométricas de la Escala de Autoeficacia para el Afrontamiento del Estrés (EAEAE). Psicothema, 20(1), 155-165.

Grau, R., Salanova, M., \& Peiro, J. M. (2012). Efectos moduladores de la autoeficacia en el estrés laboral. Apuntes de Psicología, 30(1-3), 311-321.

Guerrero, E., \& Vicente, F. (2002). Abordaje del burnout en profesionales del campo de la discapacidad. En J. N. García (Ed.), Aplicaciones de intervención Psicopedagógica (pp. 327-335). Madrid: Pirámide

Guillén, C. L., León, J. M., Gala, F. J., Sánchez-Sevilla, S., \& Ramos, E. (2005). Evaluación del estrés laboral en personal de la Administración Pública. En J. Romay \& R. García (Eds.), Psicología social y problemas sociales 4: Psicología de las Organizaciones, del Trabajo y Recursos Humanos y de la Salud (pp. 41-45). Madrid: Editorial Biblioteca Nueva

Guillén, C. L., \& Sánchez-Sevilla, S. (2012). Aspectos psicológicos asociados al proceso migratorio. En J. V. Gavidia (Ed.), Inmigración, familia y Derecho (pp. 505-517). Madrid: Marcial Pons.

Harrison, W. D. (1983). A social competence model of burnout. En B. A. Farber (Ed.), Stress and burnout in the human services professions (pp. 29-39). New York: Pergamon Press.

Karasek, R., \& Theorell, T. (1990). Healthy work: Stress, productivity, and the reconstruction of working life. New York, NY: Basic Books.

León, E., Sánchez-Sandoval, Y., Palacios, J., \& Román, M. (2010). Programa de formación para la adopción en Andalucía. Papeles del Psicólogo, 31(2), 202-210.

León, J. M., \& Avargues, M. L. (2004). Riesgos Psicosociales. En J. J. Moreno (Ed.), Manual de evaluación de riesgos laborales (pp. 283-291). Sevilla: Consejería de Empleo. Dirección General de Seguridad y Salud Laboral

Llorens, S., Schaufeli, W. B., Bakker, A., \& Salanova, M. (2007). Does a positive gain spiral of 
resources, efficacy beliefs and engagement exist?. Computers in Human Behavior, 23, 825-841. López, M., \& Marmolejo, G. (2008). La valoración psicosocial de los solicitantes de adopción. En R. Martínez \& J. M. Gómez (Eds.), La adopción de menores: Retos y necesidades (pp. 145-160). Sevilla: Asociación Andaluza de Ayuda a la Adopción y la Infancia (LLAR).

Luchman, J. N., \& González-Morales, M. G. (2013). Demands, control, and support: A meta-analytic review of work characteristics interrelationships. Journal of Occupational Health Psychology, 18(1), 37-52.

Madden-Szeszko, G. (2000). Variables contributing to teacher efficacy: An examination of burnout, affect, demographic variables, and general self-efficacy. Dissertation Abstracts International Section A: Humanities and Social Sciences, Vol 61 (3-A), Sep, 2000. pp. 881., 61(3-A). .

Maslach, C., Schaufeli, W. B., \& Leiter, M. P. (2001). Job Burnout. Annual Review. Psychology, 52, 397-422.

Mackay, C. J., \& Cooper, C. L. (1987). Occupational stress and health: Some current issue. En C. L. Cooper \& I. T. Robertson (Eds.), International Review of Industrial and Organizational Psychology (pp. 167-199). Chichester: Wiley.

Palacios, J. (2008). Manual para intervenciones profesionales en adopcion internacional. Madrid: Ministerio de Educación, Política Social y Deporte.

Peng, A. C., Schaubroeck, J. M., \& Xie, J. L. (2015). When confidence comes and goes: How variation in self-efficacy moderates stressor-strain relationships. Journal of Occupational Health Psychology, 20(3), 359-376.

Rodríguez, I., González-Morales, M. G., \& Carbonell, S. (2007). El modelo AMIG0 y la metodología PREVENLAB-PSICOSOCIAL. Aportaciones y retos en la prevención de los riesgos psicosociales. Seguridad y Salud en el Trabajo, 42, 18-25.

Sánchez-Sandoval, Y., León, E., \& Román, M. (2012). Family adaptation of internationally adopted children. Anales de Psicología, 28(2), 558-566.

Salanova, M., Grau, R., \& Martínez, I. (2005). Demandas laborales y conductas de afrontamiento: El rol modulador de la autoeficacia profesional. Psicothema, 17, 390-395.

Sánchez-Sevilla, S., Cruces, S. J., Guil, R., \& Guillén, C. L. (2012). Medidas de intervención a nivel organizacional como producto de un proyecto de innovación dirigido a la mejora del bienestar docente en el contexto de la educación superior. En C. González, D. González, J.M. Mestre \& R. Guil (Eds.), Aportaciones recientes al estudio de la motivación y las emociones (pp. 180-184). Sevilla: Fénix Editora

Sánchez-Sevilla, S., Guillén, C. L., \& León-Rubio, J. M. (2006). La autoeficacia percibida en el afrontamiento de riesgos laborales psicosociales como variable moduladora del burnout. Ansiedad y Estrés, 12(2-3), 495-504.

Vladut, C. I., \& Kallay, E. (2010). Work stress, personal life, and burnout. Causes, consequences, possible remedies. Cognition, Brain, Behavior: An Interdisciplinary Journal, 14(3), 261-280.

Yu, X., Wang, P., Zhai, X., Dai, H., \& Yang, Q. (2015). The effect of work stress on job burnout among teachers: The mediating role of self-efficacy. Social Indicators Research, 122(3), 701-708. 
